\title{
Fluoride exposure and bone quality measured using a novel ultrasonic biomarker
}

Tewodros Rango GodebO ${ }^{1, *}$, MARC JEULAND ${ }^{2}$, REDDA TEKLE-HAIMANOT ${ }^{3}$, ARTI SHANKAR ${ }^{1}$, BINIYAM ALEMAYEHU $^{3}$, GETACHEW ASSEFA ${ }^{3}$, GARY WhITFORD ${ }^{4}$, AMY WOLFE 5

${ }^{1}$ School of Public Health and Tropical Medicine, Tulane University, New Orleans, LA 70112 USA

${ }^{2}$ Sanford School of Public Policy, Duke University, Durham, NC 27708 USA

${ }^{3}$ Addis Ababa University, School of Medicine, Department of Neurology, Addis Ababa, Ethiopia

${ }^{4}$ Addis Ababa University, School of Medicine, Department of Radiology, Addis Ababa, Ethiopia

${ }^{4}$ Department of Oral Biology, College of Dental Medicine, Augusta University, Augusta, GA, USA

${ }^{5}$ Kentucky Geological Survey, University of Kentucky, KY, USA.

In this study, we assessed variation in bone quality of a population exposed to wide-ranging $\mathrm{F}^{-}$levels ( 0.3 to 15.5 $\mathrm{mg} / \mathrm{L}$ ) in drinking water, using a novel application of nonionizing ultrasonic method. We collected 871 distinct speed of sound (SOS) data from 341 subjects residing in 25 communities, aged 10-70 years (188 males and 153 females). All subjects received scans of the cortical radius and tibia, and adults over the age of 19 received an additional scan of the phalanx. Associations between $\mathrm{F}^{-}$in drinking water and 24-hour urine samples, and SOS as a measure of bone quality, were evaluated in bivariate and multivariable regressions adjusting for age, sex, BMI, smoking, and toothpaste use. We found negative associations between $\mathrm{F}^{-}$exposure and bone quality at all three bones. Adult tibial SOS showed the strongest inverse association with $\mathrm{F}^{-}$exposure, which accounted for $20 \%$ of the variance in SOS measures $(\mathrm{r}=0.45$; $\mathrm{n}=199 ; p<0.0001)$. In adjusted analysis, a $1 \mathrm{mg} / \mathrm{L}$ increase in $\mathrm{F}^{-}$in drinking water was related to a reduction of $15.8 \mathrm{~m} / \mathrm{s}$ (95\% CI: -21.3 to -10.3 ), whereas a $1 \mathrm{mg} / \mathrm{L}$ increase in $24-$ hour urinary $\mathrm{F}^{-}$(range: $0.04-39.5 \mathrm{mg} / \mathrm{L}$ ) was linked to a reduction of $8.4 \mathrm{~m} / \mathrm{s}(95 \% \mathrm{CI}:-12.7,-4.12)$ of adult tibial SOS. These results are indicative of a $\mathrm{F}^{-}$-induced deterioration of bone quality in humans, likely reflecting a combination of factors related to SOS: net bone loss, abnormal mineralization and collagen formation, or altered microarchitecture. The portable and low-cost ultrasound technique has distinct advantages over traditional x-ray technology, which is costly, difficult to use in remote areas, and exposes patients to radioactive harms. 